\title{
Performance of Non-Orthogonal Multiple Access (NOMA) with Successive Interference Cancellation (SIC)
}

\author{
Ali K. Marzook*1, Hayder J. Mohammed ${ }^{2}$, Hisham L. Swadi Roomi ${ }^{3}$ \\ ${ }^{1}$ Petroleum Engineering Department, University of Basrah, Basrah, Iraq \\ ${ }^{2}$ Alfurat Alawsat Technical University, Najaf, Iraq \\ ${ }^{3}$ Electrical engineering Department, University of Basrah, Basrah, Iraq
}

\author{
Correspondence \\ * Ali K. Marzook \\ Petroleum Engineering Department, \\ University of Basrah, Basrah, Iraq \\ Email: ali.marzook@uobasrah.edu.iq
}

\begin{abstract}
Non-Orthogonal Multiple Access (NOMA) has been promised for fifth generation (5G) cellular wireless network that can serve multiple users at same radio resources time, frequency, and code domains with different power levels. In this paper, we present a new simulation compression between a random location of multiple users for Non-Orthogonal Multiple Access (NOMA) and Orthogonal Multiple Access (OMA) that depend on Successive Interference Cancellation (SIC) and generalized the suggested joint user pairing for NOMA and beyond cellular networks. Cell throughput and Energy Efficiency (EE) are gained are developed for all active NOMA user in suggested model. Simulation results clarify the cell throughput for NOMA gained 7 Mpbs over OMA system in two different scenarios deployed users (3 and 4). We gain an attains Energy Efficiency (EE) among the weak power users and the stronger power users.
\end{abstract}

KEYWORDS: Non-orthogonal multiple access; successive interference cancellation, energy efficiency, and throughput maximization.

\section{INTRODUCTION}

Non-Orthogonal Multiple Access (NOMA) system has been recently proposed as candidate multiple access technique for $5 \mathrm{G}$ and beyond 5G systems by 3GPP in different applications [1]. NOMA system gained a wide interest as a technique of increasing the number of users that can be served simultaneously by scheduling multiple users over same spectrum resources but at different power levels [2].

Pairing of users schemes have been proposed for NOMA cellular downlink scenario with randomly deployed users [3-5]; where, the comprehensive performance of NOMA system is very affected with targeted data rates and allocated power of the deployed users. Another group of researches focused on the advantage of NOMA over Orthogonal Multiple Access (OMA) in a power allocation and fairness index in system to motivate the NOMA system as prospective system; a dynamic power allocation scheme is proposed in [6], which ensures that the individual rates for both strong and weak users in NOMA are higher than the corresponding ones in Orthogonal Multiple Access (OMA). The power allocation strategies are discussed in [7]; where they measure per-user outage probability to prove the superiority of NOMA system on OMA.

Successive Interference Cancellation (SIC) is very important toll in NOMA system which is applied at the receiver(s) for multiuser detection and decoding. To obtain of each user desired signal, the SIC processor in transmitter/receiver (uplink/downlink detection) deciphers an eminent interferences firstly and then deducts them from the superimposed signal. Since in downlink each User Equipment (UE) receives the other user's signal (the desired and interfering signals) over it channel, the superimposing of different signals with different power levels is decisive to diversifying each user signal and to perform SIC at a given UE end [8-9]. Some of authors try to improve the NOMA capacity by allocate the users into three categories (singletons, weak users, and strong users) and to derive the data rate and Signal to Interference Noise Ratio (SINR) for these categories, emphasizing the Quality of Service (QoS) of weak users [10]. 
The aforementioned studies motivated us to formulate the users allocation scenario to unification the fairness index around unity value for all users and maximization of the throughput cell of NOMA systems as a pairing optimization problem. In this paper, the performance of NOMA system is investigated in a downlink network with specific calculated positions for mobile users and Show the usefulness of this model compare with random location of users. This step is a prelude to generalizing these calculations to suggest a new uniform a cluster regions for the mobile users based on a successive values for SIC and throughput cell for all users.

The rest of the paper is organized as follows. Section II presents NOMA downlink system. Section III presents performance comparison. Sections IV and V presents the simulation results and the conclusion, respectively.

\section{NOMA DOWNLINK SYSTEM}

In NOMA downlink, we consider a standard single-cell cellular system that consists of a BS equipped with single antenna and serving $m$ users, as shown in Fig. 1

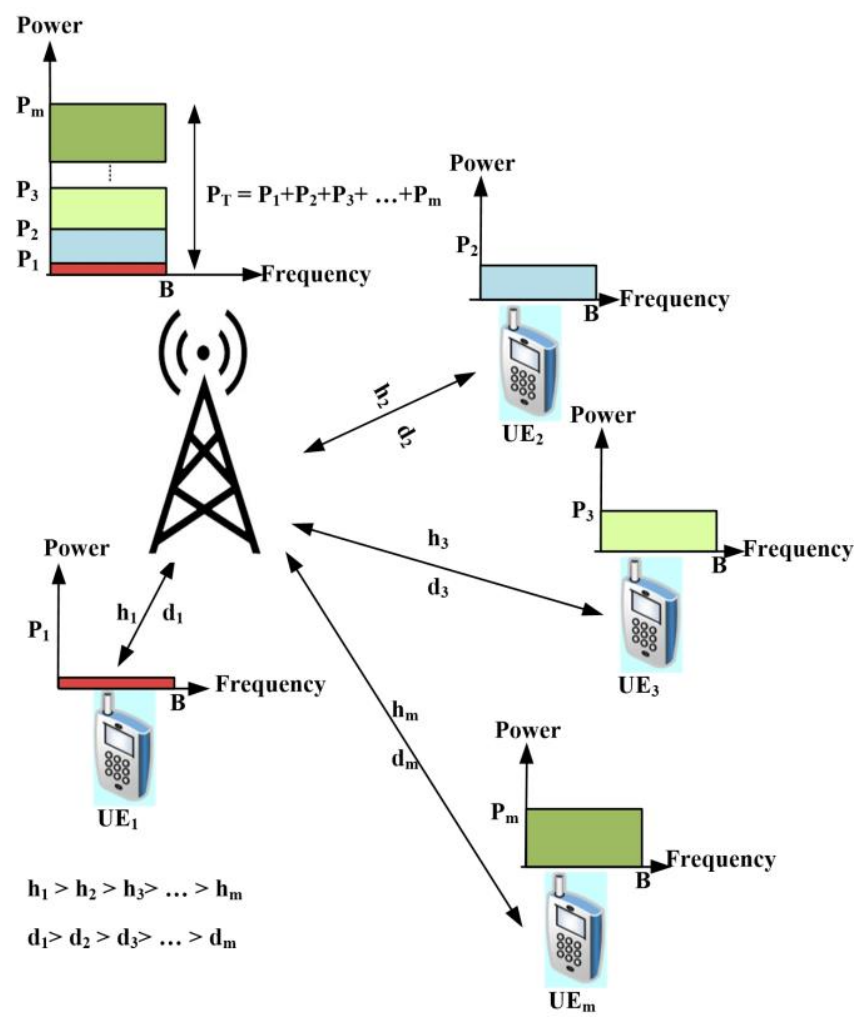

Fig. 1 NOMA downlink system

The gathering of User Equipment (UE) of all active users and SIC are employed to detect their assigned signals. Fig. 2 shows NOMA downlink system of m number of UEs end followed by SIC receivers. In the cellular network, it is supposed that the nearest user to the base station (BS) is $U E_{1}$ , while $U E_{m}$ is the farthest one.

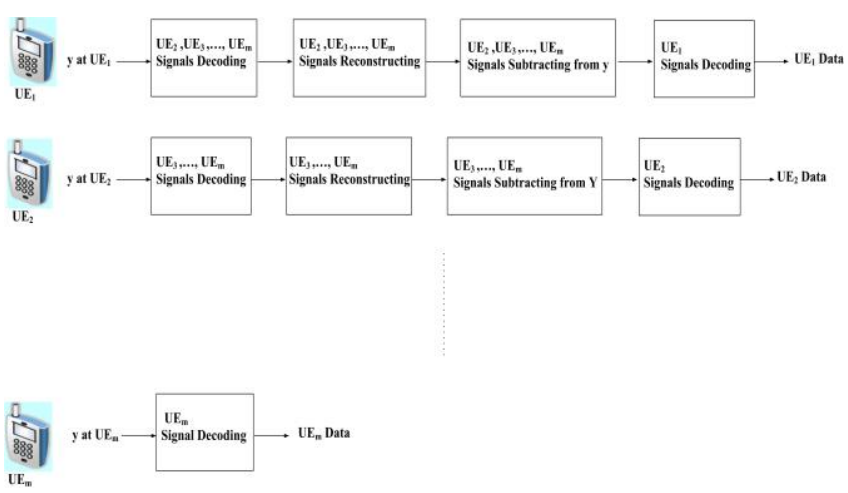

Fig.2 SCI stages for the active users

The allocated power for each user, whereas the least power assigned to the closest user and highest power assigned to the farthest and the power difference increase distributed by the most distant and the farthest among the other users, is consider the main challenge for BS. In the cellular network, each UE received the same transmitted signal that holds the data signal for the active users. Started by the farthest user which receives the high power signal, and then deducts the decoded signal from the received signal (y) to has won signal; that means the strongest user forward the signal intended for the weakest user. SIC will be carried out at the users.

Let us consider a NOMA cellular network in downlink scenarios, in which single base station has a single antenna and $\mathrm{m}$ users, each user has a single antenna too. The propagation channel between the $\mathrm{UE}_{\mathrm{i}}$ and the $\mathrm{BS}$ is presented by [3]:

$h_{i}=\frac{g_{i}}{\sqrt{1+d_{i}^{\gamma}}} \quad$ for $i=1,2, \ldots m$

Where $g_{i}$ denotes the channel gain with Rayleigh fading, $d_{i}$ represents the distance from the user to the $\mathrm{BS}$, and $\gamma$ is the path loss coefficient. The channel of the users are sorted as $\left|h_{1}\right|^{2} \leq\left|h_{2}\right|^{2} \leq \cdots \leq\left|h_{m}\right|^{2}$.

According to NOMA system, the superimposed transmitted signal which sent by the BS for all users can be expressed as:

$x(t)=\sum_{i=1}^{m} \sqrt{\alpha_{i} P_{T}} x_{i}(t)$

where $x_{i}(t)$ represents the intended data signal of OMA system, $\propto_{i}$ is the factor of power allocation for the $U_{i}$, and $\mathrm{P}_{\mathrm{i}}$ is the total average power available at the BS end.

The power allocated to for the weak and strong user $U E_{i}$ is $\mathrm{P}_{\mathrm{i}}=\propto_{\mathrm{i}} \mathrm{P}_{\mathrm{T}}$. The power allocated of each $U E_{i}$ is follows the distance of the user $U E_{i}$ from the BS; where, it distributed inversely depended on near or far the user $\mathrm{UE}_{\mathrm{i}}$ from the BS. The closest user $U_{1}$ the least power, while farthest user $\mathrm{UE}_{\mathrm{m}}$ has the strongest power. 
The individual received signal at the user end $U E_{i}$ represents as:

$y_{i}(t)=x(t) h_{i}+w_{i}(t)$

where $h_{i}$ is the factor of channel attenuation between the $\mathrm{UE}_{\mathrm{i}}$ user and $\mathrm{BS}$, and $w_{i}(t)$ denotes the received mean zero AWGN at the $\mathrm{UE}_{\mathrm{i}}$ with spectral density $N_{o}(\mathrm{~W} / \mathrm{Hz})$.

The signal of the farthest user will decoded at the first that has the most allocated power compare with the other users. While the signals for residual users will look like interference.

The signal to noise ratio (SNR) for farthest $U E_{m}$ will be indicated by [4],

$S N R_{m}=\frac{P_{m} h_{m}^{2}}{N_{o} W+\sum_{i=1}^{m-1} P_{i} h_{m}^{2}}$

where the transmission bandwidth of the system is denoted by $\mathrm{W}$.

Finally, the signal nearest user $U E_{1}$ signal will be decodes and it denoted as

$S N R_{1}=\frac{P_{1} h_{1}^{2}}{N_{o} W}$

The SNR for residual users $U E_{i}$ can written as,

$S N R_{i}=\frac{P_{i} h_{i}^{2}}{N_{o} W+\sum_{k=1}^{i-1} P_{k} h_{i}^{2}}$

The data rate achievable (the throughput) for each $\mathrm{UE}_{\mathrm{i}}$ that measured by (bps) is given by

$R_{i}=W \log _{2}\left\{1+\frac{P_{i} h_{i}^{2}}{N+\sum_{k=1}^{i-1} P_{k} h_{i}^{2}}\right\}$

In OMA, the total bandwidth system and power transmitted by $\mathrm{BS}$ are divided equally among the UEs, the data ratet for each UE in OMA is given by

$R_{i}=W_{i} \log _{2}\left\{1+\frac{P_{i} h_{i}^{2}}{N_{i}}\right\}$

Where $W_{i}$ individual bandwidth for each user and equal to $W_{i}=\frac{W}{m}$ and $N_{i}$ noise for each user and equal to $N_{i}=N_{o} W_{i}$.

The sum capacity of the systems (NOMA and OMA) can be expressed as

$R_{T}=\sum_{i=1}^{m} R_{i}$

\section{Data RATe AND POWER PerformanCES}

In this section, we demonstrate the performance of the NOMA system with OMA system.

First, we follow the cell throughput

Targeted $\mathrm{R}$ is $\max _{\propto_{i}, P t} W \log _{2}\left\{1+\frac{P_{i} h_{i}^{2}}{N+\sum_{k=1}^{i-1} P_{k} h_{i}^{2}}\right\}$

Subject to

$\mathrm{C} 1: \sum_{i=1}^{m} P_{i} \leq P_{T}$

$\mathrm{C} 2: P_{i} \geq 0, \forall i$

C3: $P_{1}<P_{2}<P_{3}<\cdots<P_{m}$

The relation between power the individual power of each user $P_{i}$ and its channel attenuation factor $\left|h_{i}\right|^{2}$ supposed to be inversely proportional, it will present by:

$P_{i} \alpha \frac{1}{\left|h_{i}\right|^{2}}$

The Constraint $\mathrm{C} 1$ denotes the total power constraint of the BS and the sum of UEs power don't exceed $P_{T}$, Constraint $\mathrm{C} 2$ ensures the has a bit of power that depend on it position from BS, and Constraint C3 ensures the power that assign by $\mathrm{BS}$ for nearest user is lower than the farthest one.

Second, the power consuming at the UEs will be exemplified as the sum of the data signal power adding to power consumed by the power amplifiers in transmitter circuits. In downlink transmitting, the consumption power in the BS can be represented as

$P_{B S T}=P_{T}+P_{c c t}$

where $P_{B S T}$ is the consumption power by BS and $P_{c c t}$ is the consumption power by amplifiers circuit at the transmitter end. Energy efficiency (EE) is defined as the sum rate over the total consumed power of the BS [6]

$E E=\frac{R_{T}}{P_{\text {total }}}=\frac{\sum_{i=1}^{m} R_{i}}{P_{\text {total }}}=\frac{m R}{P_{\text {total }}} \quad\left[\frac{\text { bits }}{\text { J.Hz }}\right]$

where $R_{T}$ is the sum of rates transmitted by UEs in terms of (bits/Hz) as mentioned earlier.

\section{SIMULATION RESULTS}

The first group of simulation results are investigated the throughput performances of the downlink NOMA system in two scenarios of users deployed (first one has a 3 users for model 1 and the second is 4 users in model 2) as cleared in Fig. 3 and Fig.5 while the second group of simulation results are investigated the energy efficiency performances for the two models that mentioned before.

The main simulation parameters are presented in Table. 1 
TABLE 1

The main simulation parameters.

\begin{tabular}{|c|c|c|}
\hline Parameters & value & Model \\
\hline $\begin{array}{c}\text { Rang of transmit } \\
\text { power }\end{array}$ & $10-80 \mathrm{dbm}$ & 1 and 2 \\
\hline The Bandwidth & $5 \mathrm{MHz}$ & 1 and 2 \\
\hline $\begin{array}{c}\text { Initial channel gain } \\
\text { of } U E_{1}\end{array}$ & $20 \mathrm{~dB}$ & \multirow{3}{*}{$1(\mathrm{~m}=3$ users $)$} \\
\hline $\begin{array}{c}\text { Initial channel gain } \\
\text { of } U E_{2}\end{array}$ & $14 \mathrm{~dB}$ & \\
\hline $\begin{array}{c}\text { Initial channel gain } \\
\text { of } U E_{3}\end{array}$ & $1 \mathrm{~dB}$ & \\
\hline $\begin{array}{c}\text { Initial channel gain } \\
\text { of } U E_{1}\end{array}$ & $20 \mathrm{~dB}$ & \multirow{4}{*}{$2(\mathrm{~m}=4$ users $)$} \\
\hline $\begin{array}{l}\text { Initial channel gain } \\
\text { of } U E_{2}\end{array}$ & $14 \mathrm{~dB}$ & \\
\hline $\begin{array}{l}\text { Initial channel gain } \\
\text { of } U E_{3}\end{array}$ & $6 \mathrm{~dB}$ & \\
\hline $\begin{array}{l}\text { Initial channel gain } \\
\text { of } U E_{4}\end{array}$ & $1 \mathrm{~dB}$ & \\
\hline$N_{o}$ & $1 \mu$ watt & 1 and 2 \\
\hline$P_{c c t}$ & $100 \mathrm{~m}$ watt & 1 and 2 \\
\hline Antennas of BS & 1 & 1 and 2 \\
\hline Antennas of UEs & 1 & 1 and 2 \\
\hline
\end{tabular}

In Fig.3; the advantage of NOMA system over OMA system is very clear in cell throughput for the whole system and each user individually in model $1 \quad(\mathrm{~m}=3)$; for example; at $P_{T}=50$ watt the NOMA system gained $6.92 \mathrm{Mbps}$ and each users gained $4.55 \mathrm{Mbps}, 1.32 \mathrm{Mbps}$, and $0.74 \mathrm{Mbps}$ for $U E_{1}$ , $U E_{2}$, and $U E_{3}$ respectively.

In Fig.4; the peak of the EE-SE curve that corresponding the derivative of the curve is where the system has the maximum energy efficiency that occur when $P_{T}=34$ watt for NOMA and OMA systems. At this power, both the systems are achieved the maximum EE, where the NOMA performance is clear has outperforms OMA system at maximum point and beyond for both EE and SE.

In Fig.5; the advantage of NOMA system over OMA system is clear too in cell throughput for the whole system and each user individually in model $2(\mathrm{~m}=4)$; for example; at $P_{T}=70$ watt the NOMA system gained $7 \mathrm{Mbps}$ and each users gained $4.83 \mathrm{Mbps}, 1.03 \mathrm{Mbps}, 0.36 \mathrm{Mbps}$, and 0.67 Mbps for $U E_{1}, U E_{2}, U E_{3}$, and $U E_{4}$ respectively.

For model 2, the system has the maximum energy efficiency that occur when $P_{T}=36 P_{T}=34$ watt for NOMA and OMA systems respectively. The NOMA performance is clear has outperforms OMA system at maximum point and beyond for both EE and SE that was clear in Fig. 6.

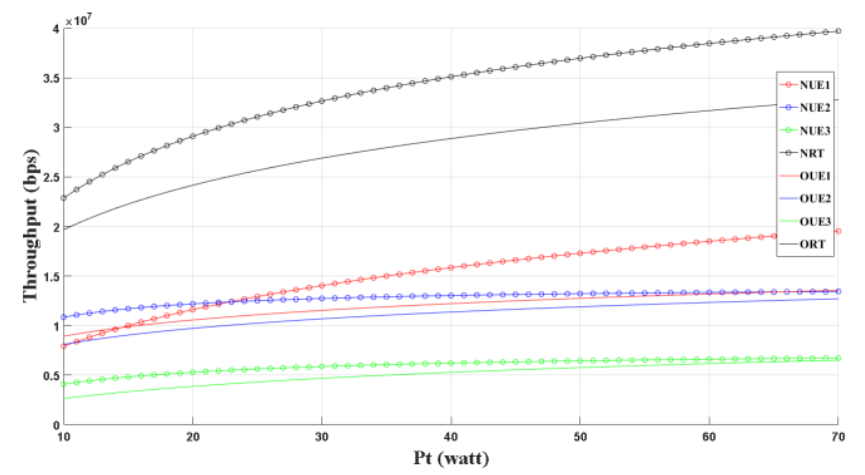

Fig. 3 Cell throughput rates for downlink NOMA and OMA systems at $\mathrm{m}=3$.

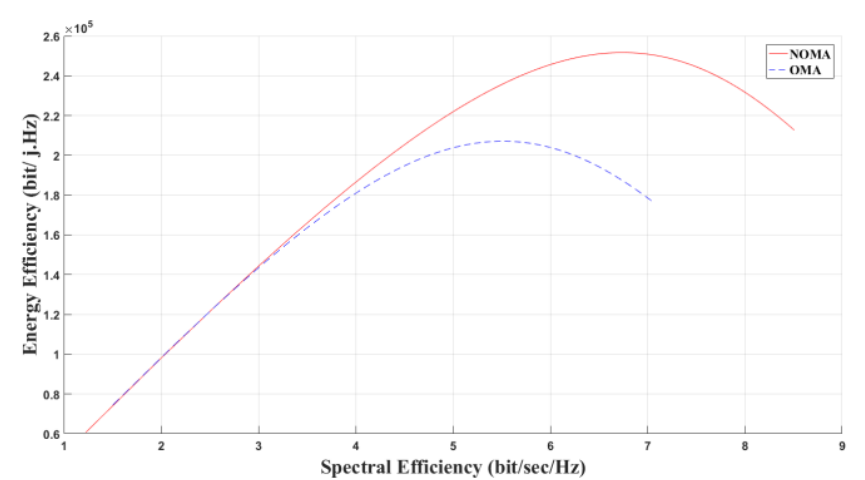

Fig.4 EE-SE curves for NOMA and OMA systems at $\mathrm{m}=3$.

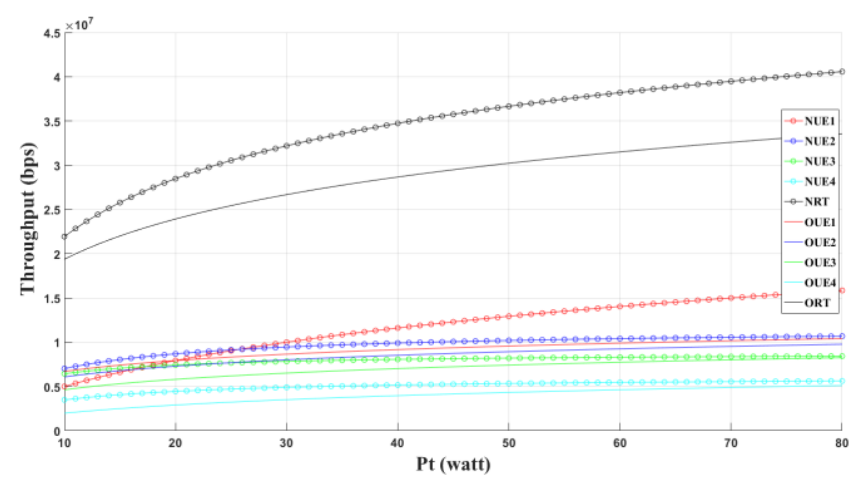

Fig. 5 Cell throughput rates for downlink NOMA and OMA systems at $\mathrm{m}=4$.

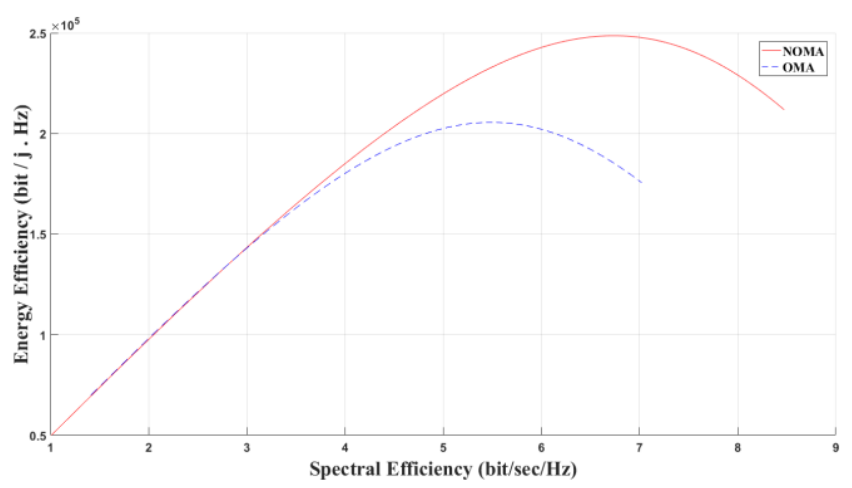

Fig.6 EE-SE curves for NOMA and OMA systems at $\mathrm{m}=4$. 


\section{CONCLUSIONS}

In this paper, the throughout performance in NOMA and OMA systems have been examined. We compare its performance for two different scenarios of deployed users ( 3 users for model 1 and 4 users for model 2). Through the simulation the highest throughput cell can be achieved at specific range of total base station power. Based on the utility of NOMA system can be suggested as a potential technique for $5 \mathrm{G}$ mobile communication system. There are many direction for future work like a power allocation of each user and gathering them in clusters. SIC techniques will enhance the performance due to can consider it as a hot area research.

\section{CONFLICT OF INTEREST}

The authors have no conflict of relevant interest to this article.

\section{REFERENCES}

[1] J. Choi, "Minimum power multicast beamforming with superposition coding for multiresolution broadcast and application to NOMA systems," IEEE Trans. Commun., vol. 63, no. 3, pp. 791-800,2015.

[2] Z. Ding, X. Lei, G. K. Karagiannidis, R. Schober, J. Yuan, and V. K. Bhargava, "A survey on non-orthogonal multiple access for 5g networks: Research challenges and future trends," IEEE Journal on Selected Areas in Communications, vol. 35, no. 10, pp. 2181-2195, 2017.

[3] Zhiguo Ding, Zheng Yang, Pingzhi Fan, and H. Vincent Poor, "On the Performance of Non-Orthogonal Multiple Access in 5G Systems with Randomly Deployed Users". IEEE Signal Processing Letters, vol. 21, no. 12, pp. 1501 1505, 2014.

[4] Peng Xu, Zhiguo Ding, Xuchu Dai and H. Vincent Poor "NOMA: An Information Theoretic Perspective. Multiple Access Techniques for 5G Wireless Networks and Beyond, Springer, pp 167-193". 2015

[5] Z. Ding, P. Fan, and V. Poor, "Impact of User Pairing on 5G NonOrthogonal Multiple Access Downlink Transmissions," IEEE Trans. Veh. Technol., vol.65, no. 8, pp. 6010 - 6023, 2016.

[6] E. Hossain, M. Rasti, H. Tabassum, and A. Abdelnasser, "Evolution toward 5G multi-tier cellular wireless networks: An interference management perspective," IEEE Wireless Communications, vol. 21, no. 3, pp. 118-127, 2014.

[7] M. F. Sohail, C. Y. Leow, and S. Won, "Non-orthogonal multiple access for unmanned aerial vehicle assisted communication," IEEE Access, vol. 6, no. 1, pp. 22716 22727, 2018.

[8] M. Saideh, Y. Alsaba, I. Dayoub, and M. Berbineau, "Joint interference cancellation for multi-carrier modulation-based non-orthogonal multiple access," IEEE Commun. Lett., vol. 23, no. 11, pp. 2114-2117, 2019.

[9] K. Higuchi and A. Benjebbour, "Non-orthogonal multiple access (NOMA) with successive interference cancellation for future radio access," IEICE Transactions on Communications, vol. 98, no. 3, pp.403-414, 2015.
[10] S. Dhakal, P. A. Martin, and P. J. Smith, "Noma with guaranteed weak user qos: Design and analysis," IEEE Access, vol. 7, no. 2, pp. 32884-32896, 2019. 